\title{
Abdominal Wall Mass: An Unusual Complication of a Ventral Hernia Repair
}

\author{
Sang Lee ${ }^{\mathrm{a}}$, Paul Hanna ${ }^{\mathrm{b}}$, Daniel Barbash ${ }^{\mathrm{b}}$, Manrique Guerrero ${ }^{\mathrm{b}}$, \\ Jamshed Zuberi ${ }^{b}$, Scott Wessner ${ }^{\text {b, c }}$
}

\begin{abstract}
An abdominal wall mass can arise from a large number of etiologies, but it has not been reported as a complication of a ventral hernia repair. The most common complication of a hernia repair is recurrence. Here, we report an unusual case of a 47-year-old male who presented with a benign abdominal mass 12 years after undergoing a ventral hernia repair, and describe our recommended surgical management.
\end{abstract}

Keywords: Abdominal wall mass; Ventral hernia repair; Ventral hernia repair complication

\section{Introduction}

A ventral hernia repair (VHR) is one of the most common elective procedures performed by general surgeons. Reported complications of VHR include recurrence [1], persistent posterior seroma [2], and mesh erosion from a displaced mesh causing small bowel obstruction [3]. Abdominal mass following a VHR is rarely reported. In this unusual case we discuss a case of a 47-year-old male, with no significant past medical history who presented with a rapidly growing and hemorrhaging mass of fibroadipose tissue in the abdominal wall at the location of his hernia repair site 12 years prior.

\section{Case Report}

A 47-year-old male with no significant past medical history presented to the emergency room with gradually worsening left abdominal pain and no radiation for the past 2 months. No relieving or exacerbating factors were observed. Patient denied nausea, emesis, or constipation. Patient denied any history of

Manuscript submitted October 17, 2018, accepted October 25, 2018

aSaint George's University, West Indies, Grenada

bSt. Joseph's Regional Medical Center, Paterson, NJ, USA

${ }^{\mathrm{c} C}$ Corresponding Author: Scott Wessner, 703 Main Street, Paterson, NJ 07503,

USA. Email: scottwessner@gmail.com

doi: https://doi.org/10.14740/jcs357w trauma to the abdomen; however, patient did have a VHR 12 years ago, and it was unknown whether mesh was used. Family history was noncontributory, and he had no known drug allergies. Patient also denied any toxic habits. On physical examination, he had a temperature of $36.6^{\circ} \mathrm{C}$, pulse of 89 , blood pressure of 133/94 mm Hg, respiratory rate of 20, and oxygen saturation of $100 \%$ on room air. No lymphadenopathy or signs of oral manifestations of disease were observed. Abdominal exam demonstrated a large nonpulsatile, nonmobile mass in the left lower quadrant of his abdomen with normal bowel sounds.

Laboratory data showed white blood cell count of 9.0k cells $/ \mathrm{mm}^{3}$ with $59 \%$ neutrophils, and electrolytes and coagulation parameters were within normal limits.

Computed tomography (CT) scan of the abdomen 4 years prior to presentation, but after the VHR revealed an $11-\mathrm{cm}$ long extraperitoneal soft tissue density along the left hemiabdomen (Fig. 1). CT scan of the abdomen was repeated upon presentation and revealed the same retroperitoneal soft-tissue density along the left hemi-abdomen measuring approximately $10 \times 4.8 \mathrm{~cm}$. Patient was discharged in stable condition and planned for a CT-guided biopsy as an outpatient. This biopsy showed benign fibroadipose tissue with focal scattered chronic inflammation without malignancy. A few days following the biopsy, which was 1 month after the initial emergency room visit, the patient returned with worsening abdominal pain, nausea and vomiting. CT of the abdomen was repeated and showed that the mass had increased in size to $17 \times 5.8$ $\mathrm{cm}$ with a slight increase in the hemorrhage associated with the mass (Fig. 2). Patient was thought to have an acute hemorrhage from the biopsy. Patient subsequently had magnetic resonance imaging (MRI) for more detailed findings of the mass and to rule out an abdominal wall sarcoma (Fig. 3). With a hemoglobin drop from $12 \mathrm{~g} / \mathrm{dL}$ to $9 \mathrm{~g} / \mathrm{dL}$, it was decided to take him to the operating room. At exploration, patient had excision of the anterior wall mass and complex VHR with component separation and mesh (Fig. 4). A left-sided oblique incision was made encompassing the overlying scar, which was excised (Fig. 4a). A plane between the medial part of the mass and peritoneum was obtained allowing for separation of the mass from the peritoneum, both inferiorly and laterally. The mass extended from the costal margin superiorly to the iliac crest inferiorly. Ultimately, a large $14 \times 11 \mathrm{~cm}$ defect over the peritoneum was excised. Polypropylene mesh was secured medially to the rectus fascia, superiorly just above the costal margin, posteriorly along the gutter, and inferiorly to the iliac 

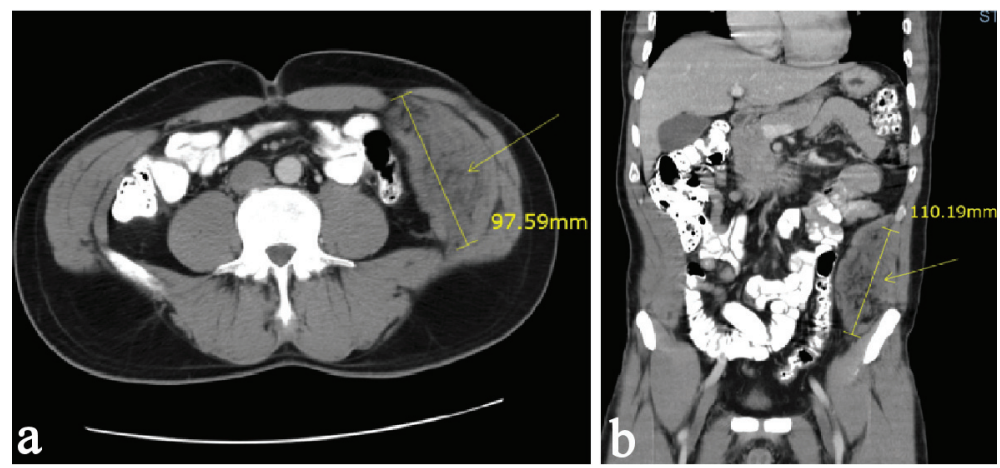

Figure 1. $(a, b)$ CT scan of the abdomen and pelvis demonstrating an 11-cm long extraperitoneal soft tissue density along the left hemi-abdomen.
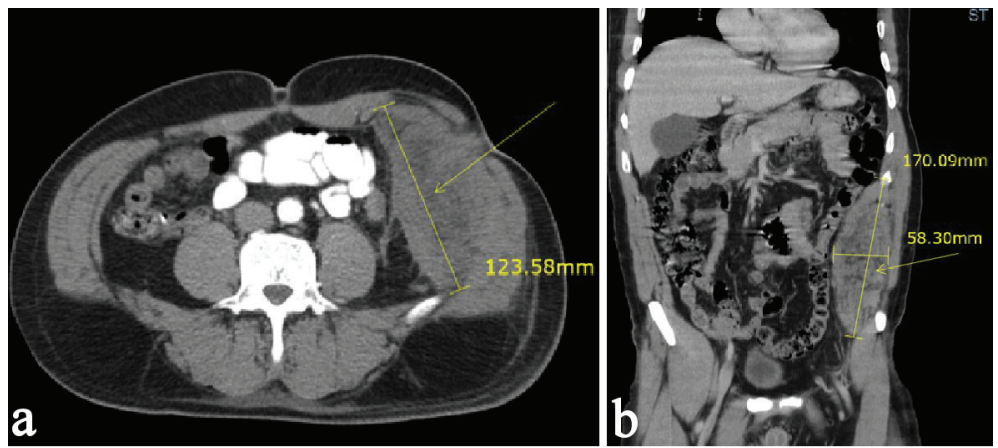

Figure 2. (a, b) CT scan of the abdomen and pelvis after CT-guided biopsy demonstrating a mass increased in size of $17 \times 5.8$ $\mathrm{cm}$ with a slight increase in the hemorrhage associated with the left flank/ abdominal wall mass.

crest. The specimen was examined by pathology and found to be the same as the biopsy result of fibroadipose tissue with extensive hemorrhagic fibrinoid necrosis. Patient tolerated the procedure without complications and was discharged home several days later.

\section{Discussion}

Abdominal wall masses in adults can be divided into five gen- eral causative categories: neoplastic, inflammatory/infective, traumatic, iatrogenic and congenital [4]. In this case, although the biopsy shows fibroadipose tissue, we were still concerned that the mass could be a sarcoma or other malignancy. Patient's associated pain was probably caused by the compression of surrounding neural structures [4]. A mass of fibroadipose tissue many years following a ventral hernia repair has not been well documented in literature. Our literature review identified case reports discussing the following less common complications of hernia repair: persistent posterior seroma [2] and mesh
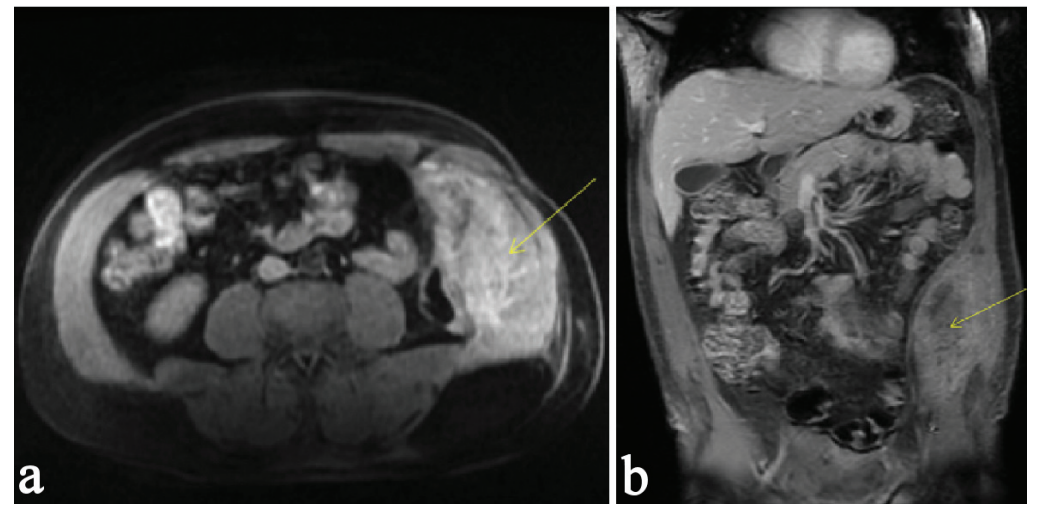

Figure 3. $(a, b) M R I$ of the abdomen and pelvis demonstrating a hemorrhage associated in the left lateral abdominal wall mass. The hyperintensity shown on the MRI marked by the arrowhead indicates the presence of a hemorrhage. 

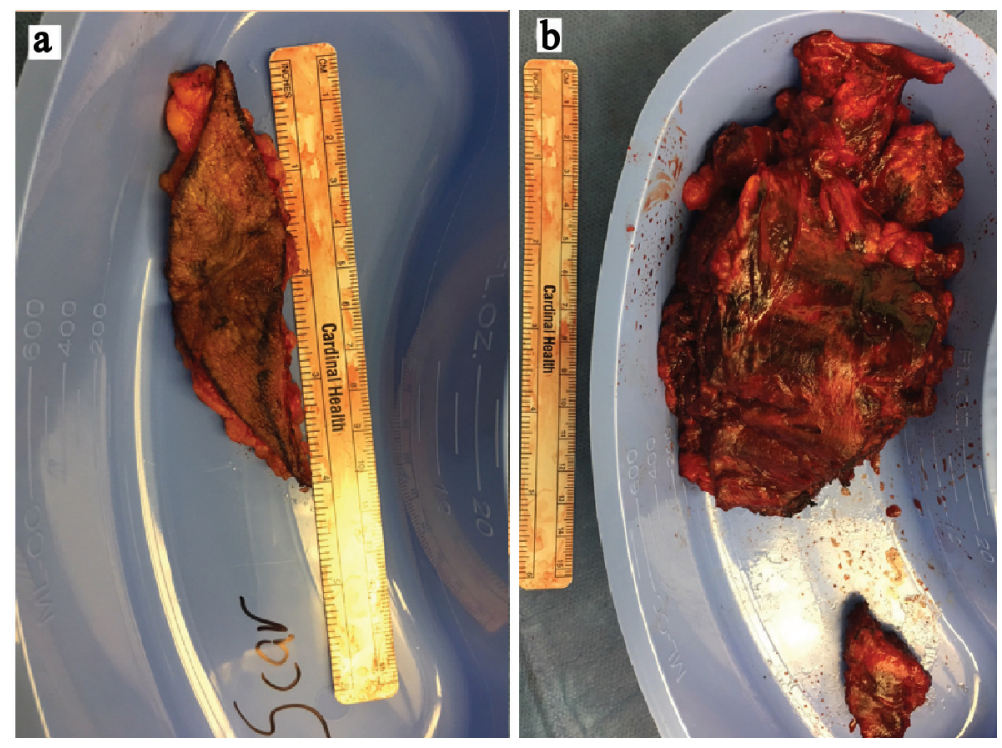

Figure 4. (a, b) Abdominal wall mass originating from hernia repair incision scar.

erosion from a displaced mesh causing small bowel obstruction [3]. However, we could not find any case reports on growing and hemorrhaging masses of fibroadipose tissue in the abdominal wall at the location of a previous hernia repair site. Pathologic examination of these masses is important to differentiate a benign fibroadipose tissue from other neoplasms, such as sarcoma, which can present similarly clinically and on radiographic imaging. Recent studies have highlighted that experienced examiners are able to achieve high diagnostic accuracy in distinguishing between lipomas and atypical lipomatous tumor/well-differentiated liposarcomas on the basis of MRI characteristics, particularly considering size, margins, homogeneity of the signal and the presence of septa or nodules [5]. Close follow-up of this patient with an appropriate imaging modality will be beneficial because there is a lack of understanding on the origin of the mass. Patient underwent multiple diagnostic workups (i.e., CT images, CT-guided biopsy, and MRI) and a procedure to excise the mass. Histopathology confirmed the diagnosis of benign fibroadipose tissue. Despite all the clinical and histopathological investigations the patient underwent, we were not able to find an etiology of this growing abdominal wall mass. Following up on literature similar to this case in the future will allow us to understand the link between an abdominal wall mass and the history of herniorrhaphy. We present this case in an effort to demonstrate an unusual complication of a VHR.

\section{Conflict of Interest}

There is no conflict of interest in this project.

\section{References}

1. Kokotovic D, Bisgaard T, Helgstrand F. Long-term recurrence and complications associated with elective incisional hernia repair. JAMA. 2016;316(15):1575-1582.

2. Xue TM, Tao LD, Zhang J, Zhang PJ. Mesh erosion causes small bowel obstruction: a rare complication of laparoscopic inguinal hernia repair: case description and review of literature. Hepatogastroenterology. 2015;62(137):55-58.

3. Watson HI, Saunders AJ. Fibrolipomas masquerading as abdominal hernias. BMJ Case Rep. 2013.

4. Stirler VMA, de Haas RJ, Raymakers J, Rakic S. Persistent posterior seroma after laparoscopic repair of ventral abdominal wall hernias with expanded polytetrafluoroethylene mesh: prevalence, independent predictors and detached tacks : Retrospective review. Hernia. 2018;22(2):285-291.

5. Coran A, Ortolan P, Attar S, Alberioli E, Perissinotto E, Tosi AL, Montesco MC, et al. Magnetic resonance imaging assessment of lipomatous soft-tissue tumors. In Vivo. 2017;31(3):387-395. 